\title{
Crossroads: advanced guidance through an aortic coarctation by fusion imaging in transfemoral TAVR after aorto-aortic bypass
}

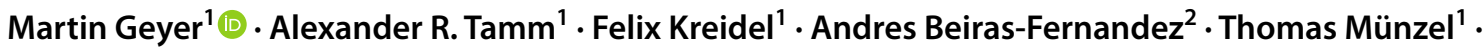 \\ Ralph Stephan von Bardeleben ${ }^{1}$
}

Received: 5 February 2021 / Accepted: 22 March 2021 / Published online: 29 March 2021

(c) The Author(s) 2021

Keywords Aortic stenosis $\cdot$ TAVI $\cdot$ Aortic coarctation $\cdot$ Fusion imaging $\cdot$ Multidisciplinary heart team

A 78-year-old male patient with symptomatic aortic valve stenosis and history of surgical therapy of a preductal aortic coarctation by an aorto-aortic bypass was admitted to our Heart Center (Fig. 1; A: 3D-reconstruction from CT/B: fluoroscopy/C: fusion imaging; elongated native aortic arch (a) with coarctation (arrow), and aorto-aortic bypass (b)). Echocardiography and CT scan showed a degenerated and severely calcified bicuspid aortic valve (Type I LR, according to Sievers classification [1], D). Severe annular calcification was favoring the selection of a self-expandable prosthesis. Intricacy of vascular access for transcatheter aortic valve replacement (TAVR) was relevantly increased due to the patient's special anatomy with an intact aortic bypass graft bearing a large appositional thrombus $(* *, E)$, whereas the patent native aortic arch was stenotic and elongated in kind of a "double-z" shape with two stenotic segments (smallest vessel diameter $9 \mathrm{~mm}$ in a $135^{\circ}$ curve directly distal to the ostium of the left carotid artery, see arrow in A and red color markers in C). As an alternative subclavian access was unfavorable due to angulation and vessel diameter, we decided for the "long way" in kind of transfemoral approach via the native kinked aortic arch.

Implementation of fusion imaging of both fluoroscopy and the screening CT scan entailed safe guidance of the stiff wire (asterisk, C; shape of the coarctation's angulation only moderately affected by the guidewire) through the aortic coarctation (see also supplemental video). A Medtronic EvolutPro® 29 mm (Medtronic, Minneapolis, MN, USA) TAVR-prosthesis (\#, F) was implanted without any problems after pre-dilatation by balloon-valvuloplasty (peak-to-peak gradient $>100 \mathrm{mmHg}$ ) with a good result of excellent hemodynamics and mild paravalvular leakage.

Our case demonstrates that advanced guidance by fusion imaging can facilitate safe TAVR-procedures even in uncommon and complicated anatomies.
Martin Geyer

martin.geyer@unimedizin-mainz.de

1 Department of Cardiology, Cardiology I, University Medical Center of the Johannes Gutenberg-University Mainz, Langenbeckstr. 1, 55131 Mainz, Germany

2 Department of Cardiothoracic and Vascular Surgery, University Medical Center of the Johannes Gutenberg-University, Langenbeckstr. 1, 55131 Mainz, Germany 


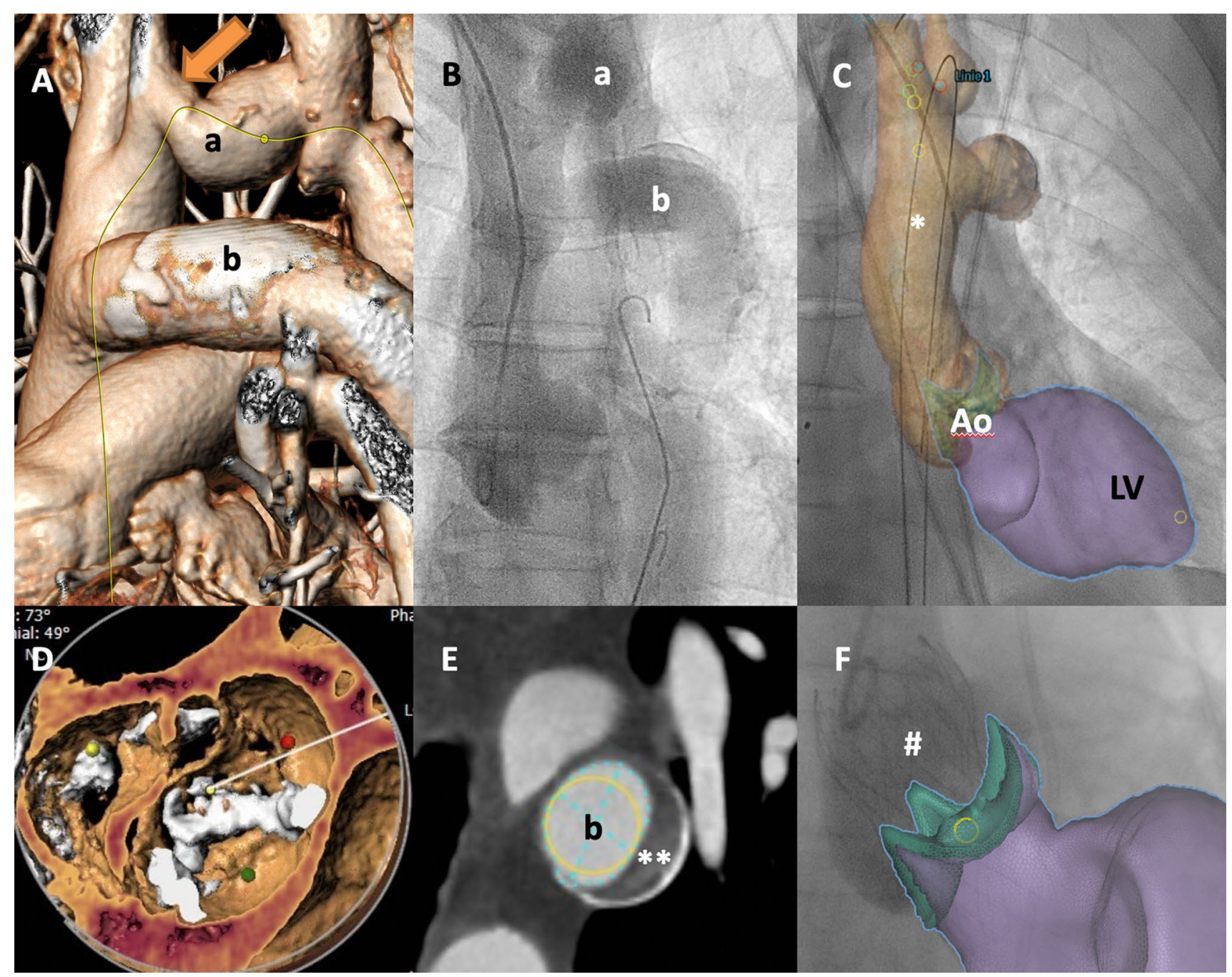

Fig. 1 A CT-reconstruction of "double" aortic arch: elongated, stenotic native aortic arch (a) and aorto-aortic bypass (b), arrow indicating smallest vessel diameter of the coarctation. B Fluoroscopy (via radial pigtail-catheter, tip in non-coronary sinus): native aortic arch (a) and aorto-aortic bypass (b). C Fusion imaging (LV, left ventricle; Ao, aortic valve), stiff wire $(*)$ guided by real-time fusion imaging

Supplementary Information The online version contains supplementary material available at https://doi.org/10.1007/s12928-021-00772-9.

Funding Open Access funding enabled and organized by Projekt DEAL.

\section{Declarations}

Conflict of interest Ralph Stephan von Bardeleben reports having received consultancy and lecture honoraria from Abbott Structural Heart, Boehringer Ingelheim, Cardiac Dimensions, Edwards Lifesciences, GE Health Systems and Philips Healthcare. All other authors declare no conflicts of interest. through native stenotic aortic arch (color markers indicating stenotic segment). D CT-reconstruction of native aortic valve stenosis (n. b. calcified fusion of left and right coronary cusp/bicuspid aortic valve Typ I LR). E CT-reconstruction of aorto-aortic bypass (b) with appositional thrombus $(* *)$. F Fusion imaging: result aortography showing self-expanding TAVR-prosthesis (\#) in situ

Open Access This article is licensed under a Creative Commons Attribution 4.0 International License, which permits use, sharing, adaptation, distribution and reproduction in any medium or format, as long as you give appropriate credit to the original author(s) and the source, provide a link to the Creative Commons licence, and indicate if changes were made. The images or other third party material in this article are included in the article's Creative Commons licence, unless indicated otherwise in a credit line to the material. If material is not included in the article's Creative Commons licence and your intended use is not permitted by statutory regulation or exceeds the permitted use, you will need to obtain permission directly from the copyright holder. To view a copy of this licence, visit http://creativecommons.org/licenses/by/4.0/. 


\section{Reference}

1. Sievers HH, Schmidtke C. A classification system for the bicuspid aortic valve from 304 surgical specimens. J Thorac Cardiovasc Surg. 2007;133:1226-33.
Publisher's Note Springer Nature remains neutral with regard to jurisdictional claims in published maps and institutional affiliations. 